\title{
Simulated Decrab Maneuver: Evaluation with a Pilot Perception Model
}

\author{
E.L. Groen ${ }^{1}$, \\ TNO Human Factors, Soesterberg, The Netherlands \\ M.H. Smaïli' ${ }^{2}$, \\ National Aerospace Laboratory, Amsterdam, The Netherlands \\ and \\ R.J.A.W. Hosman ${ }^{3}$ \\ AMS Consult, Delfgauw, The Netherlands
}

\begin{abstract}
In this simulator study eleven pilots rated their motion perception during a series of decrab maneuvers of a twin-engine passenger aircraft. Platform yaw, sway, and roll motion were varied independently to examine their relative contribution to the pilots' judgements. In one set of conditions, the washout algorithms were bypassed so as to reproduce unfiltered aircraft motion. This was compared with washout-filtered motion in another set of conditions. Moreover, the effect of visual cues was studied by testing the unfiltered motion cues once under simulated VMC, and once under IMC. The results show that the simulation of heading alignment was positively affected by platform sway and roll, and also by the visual stimulus. Platform yaw was poorly recognized, and remained under the perceptual threshold in the presence of platform sway. Interestingly, unfiltered sway motion was perceived as too strong, even though the simulator workspace required downscaling to $70 \%$ of the actual aircraft motion. Finally, the subjective data was used to validate our human motion perception model. Due to the fact that the model does not yet account for the observed interaction between sway motion and the perceptual threshold for yaw, the model output did not quantitatively correlate with the magnitude ratings. However, a multiple regression analysis showed that, qualitatively, the model did predict the way pilots interpreted the platform motion. We conclude that better understanding of perceptual thresholds in a flight simulator setting is necessary to enable quantitative analysis of the effectiveness of ground-based motion cues.
\end{abstract}

\section{Nomenclature}

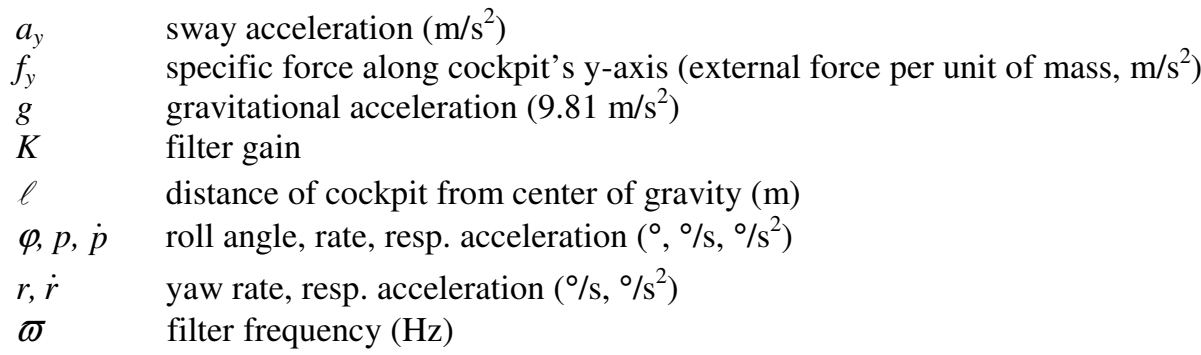

\footnotetext{
${ }^{1}$ Aerospace Physiologist, Education and Training Department, P.O.Box 23, 3769 ZG Soesterberg.

2 Aerospace Engineer, Training, Human Factors and Cockpit Operations Department, P.O. Box 90502, 1006 BM Amsterdam.

${ }^{3}$ Pilot Engineer, Dijkgraafstraat 26, 2645 KN Delfgauw, AIAA Member.
} 


\section{Introduction}

$M^{0}$

ODELS on pilot control and perception can be helpful in the off-line assessment of motion requirements for motion-critical flight tasks ${ }^{1,2}$. Several authors have used a vestibular model, composed of transfer functions of the semicircular canals and otoliths, to optimize the correspondence between the pilot's sensory responses in the simulator and in the real aircraft ${ }^{3-6}$. At TNO we developed a human motion perception model that also includes multisensory interactions accounting for the central interpretation of sensory cues by the central nervous system (CNS) ${ }^{7}$. The input for the model is a six degrees-of-freedom (DoF) time history of aircraft-, or simulator motion. The output gives the corresponding time histories of perceived self-motion and orientation (i.e. attitude). In two previous papers we reported on the effectiveness of the model in evaluating the simulation of a takeoff run $^{8}$, and the simulation of the rotation and first segment $\mathrm{climb}^{9}$, respectively. The required psychophysical data were obtained in two flight simulator studies ${ }^{10,11}$. This new study was designed to validate the model for an asymmetric flight maneuver, which is more motion-critical than both previous symmetric maneuvers.

A unique element in the simulator experiment was that, by pre-programming the platform motion, we were able to reproduce the actual aircraft motion for the specific maneuver without transformation by the washout algorithms. The rationale for this was that washout algorithms usually apply to the whole flight envelope, which results in rather conservative simulator motion. This would make it difficult to determine the exact motion aspects that are most useful to the pilot's perception or control behavior.

The selected asymmetric maneuver was an early decrab during the final approach of a crosswind landing. This maneuver aligns the aircraft's heading with the runway before touch down. In coordinated flight before decrab, the aircraft is pointed a few degrees into the wind. The pilot applies rudder to align the aircraft with the centerline, thereby introducing a slip angle. At the same time, the upwind wing is lowered so as to prevent downwind drift. Banking the aircraft tilts the lift vector toward the upwind side, providing a force that counteracts the effect of sideslip on the fuselage. In terms of motion cues acting on the pilot, the decrab thus consists of a combination of yaw and roll motion. In addition, with the cockpit of transport aircraft located at a considerable distance in front of the center of gravity, yaw acceleration automatically generates sway acceleration at the pilot's position, according to:

$$
a_{y}=\dot{r} \ell
$$

This leads to the research question whether both motion cues, yaw or sway, are equally useful to the pilot. Moreover, the attitude change in roll causes a gravity component $g_{y}$ to project along the pilot's y-axis, which may be confused with the sway acceleration $a_{y}$ taking place in the same plane. The TNO motion perception model takes this perceptual ambiguity between linear acceleration and tilt into account. This addresses the second research question, whether the motion perception model explains how these ambiguous motion cues, sway or roll, are being interpreted. These two research questions can be formulated as two hypotheses:

1. The perceived motion during a simulated decrab maneuver is equally dependent of the yaw and sway motion.

2. The pilots' judgments of alignment motion are better predicted by the output of the motion perception model than by the motion stimulus settings.

\section{Flight simulator experiment}

\section{A. Simulator facility}

The simulator experiment took place at the National Aerospace Laboratory NLR in Amsterdam. Since the new NLR facility (GRACE) with a civil cockpit was not available at the time of the experiment, the Generic Fighter Operations Research Cockpit Environment (GFORCE) simulator was employed. This facility features a modifiable modular F-16 fighter cockpit placed inside a 17ft dome projection system. The 6-Degree-of-Freedom (DoF) motion platform consists of a hydraulic hexapod, manufactured by Rexroth-Hydraudyne. The motion platform is characterized by a high bandwidth $\left(45^{\circ}\right.$ phase lag at $\left.4 \mathrm{~Hz}\right)$ and movements of $\pm 29^{\circ}$ pitch, $\pm 30^{\circ}$ roll, $\pm 41^{\circ}$ yaw, total $2.1 \mathrm{~m}$ heave, $\pm 1.4 \mathrm{~m}$ sway and $+1.7 /-1.3 \mathrm{~m}$ surge (Figure 1 ). The visual system consisted of a three channel Evans \& Sutherland ESIG-3000 computer image generation system. The field-of-view of the out-the-window image amounted to $140^{\circ}$ (horizontal) $\times 110^{\circ}$ (vertical). Although the field-of-regard can be enlarged by the head-slaved projector, this option was not used in this experiment, and pilots were instructed to look straight ahead. 

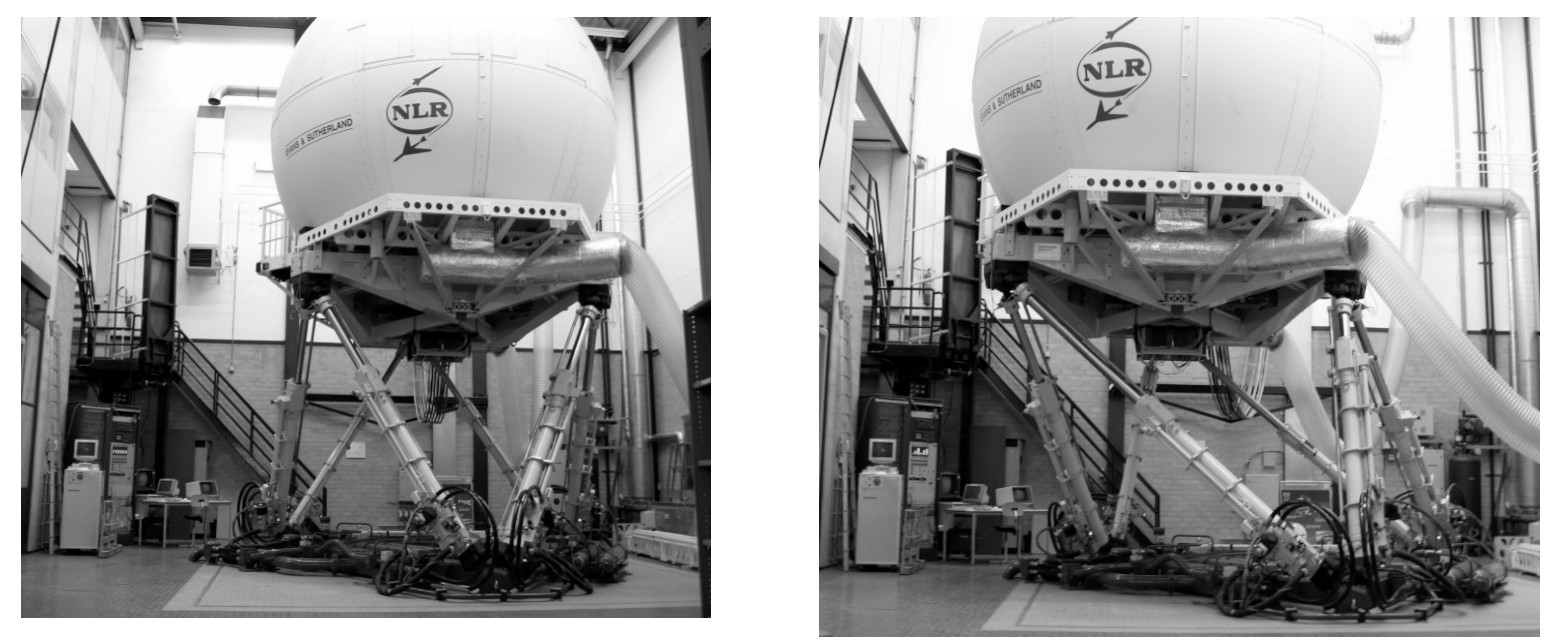

Figure 1. NLR Research flight simulator (GFORCE) with the six-DoF motion system in its neutral position (left), and pre-positioned for simulation of unfiltered motion (right).

The visual database featured the San Francisco International Airport and surrounding area. In the experiment only the following basic flight instruments were functional: attitude indicator, altimeter, airspeed and heading indicator. Cockpit and visual system representation during the simulated decrab maneuver, as observed by the pilot, is depicted in Figure 2. Although no sound cues were simulated, the noise in the cockpit effectively masked all platform motion.
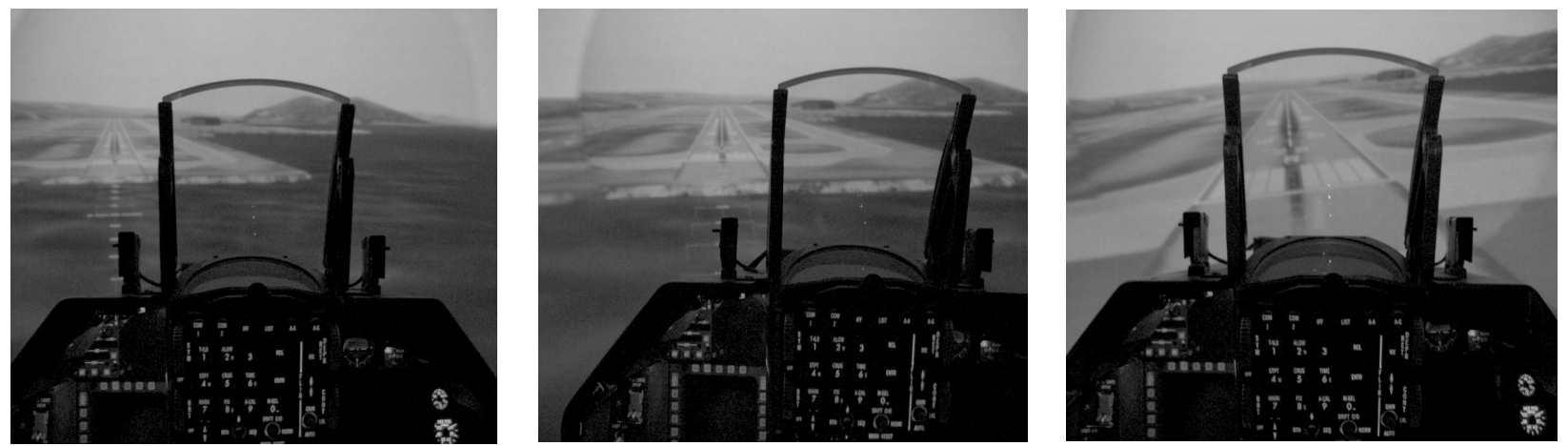

Figure 2. Three time-lapse images from inside the cockpit showing the sequence of the simulated decrab maneuver (from left to right).

\section{B. Pilots}

Eleven professional airline pilots volunteered in this study. They all were experienced on a twin-engine 100 passenger aircraft such as a Fokker F100 (mean age 41 years, 7000 flight hours). For two reasons, we decided not to give the pilots an active flight task. First, as airline pilots they were unfamiliar with the layout of the fighter cockpit. And second, by having the pilots passively monitor an autoland decrab, we were able to carefully control the motion profile, and expose all pilots to exactly the same inertial motion stimulus.

\section{Aircraft model}

The aircraft model used in this study was developed in Matlab ${ }^{\circledR}$ Simulink ${ }^{\circledR}$, and comprised a simplified model of the autoland decrab of a generic, twin-engine 100 passenger aircraft. The model was simplified and adapted from an F100 autoland decrab. The purpose of this simplification was to adjust some model parameters so that the essential motion cues would theoretically be above the perception threshold. Although different criteria for 
perceptual thresholds may be used, in flight simulation it is the "indifference" threshold that is considered most relevant ${ }^{13}$. For yaw rate the indifference threshold is reported to be $0.5 \%$, and for linear acceleration it is on the order of $0.1 \mathrm{~m} / \mathrm{s}^{2}$. The distance between the cockpit and the center of gravity used here amounted to $15 \mathrm{~m}$, so that the yaw acceleration about $1.2^{\circ} / \mathrm{s}^{2}$ was accompanied by $0.3 \mathrm{~m} / \mathrm{s}^{2}$ of sway acceleration during the alignment phase (see [1]). The output of the simplified aircraft model is plotted in Figure 3 for linear acceleration along the pilot's y-axis, and angular motion (yaw and roll). The peak in the lateral acceleration at 5s results from [1]. Yaw velocity reached a maximum of $2 \%$, which is about four times the above-mentioned indifference threshold.

\section{Experimental design}

In total there were 24 different conditions, as schematically depicted in Figure 4. Simulator motion consisted of yaw, sway, and roll motion. Each motion cue could be either present or absent, giving eight combinations of three motion cues $(2 \times 2 \times 2)$. These eight different motion profiles were input to the motion platform after transformation by the washout software in one set of conditions (Washout ON). Classical washout was applied with second-order high-pass filters for the onset cue of sway motion $(\varpi=0.25, \mathrm{~K}=0.5)$, and for the onset cues of yaw and roll angular motion ( $\varpi=0.25, \mathrm{~K}=1$ and $\mathrm{K}=0.6$, respectively). Tilt co-ordination for sustained sway motion was achieved with a second-order low-pass filter ( $\varpi=1, \mathrm{~K}=0.6$ ). Damping coefficients of all filters were set at one. In another set of conditions (Washout OFF), the motion output from the aircraft model was sent directly to the motion platform, thus bypassing the washout software. Using pre-programmed motion profiles allowed us to pre-position the simulator in its outmost left or right position (Figure 1, right), so as to utilize the maximum linear travel of $2.5 \mathrm{~m}$. By simulating both linear and rotational motion aspects of the decrab maneuver unfiltered, the temporal behavior of the aircraft motion was unaltered. The only compromise was a $30 \%$ reduction in amplitude, which was necessary to stay within the simulator's linear workspace.
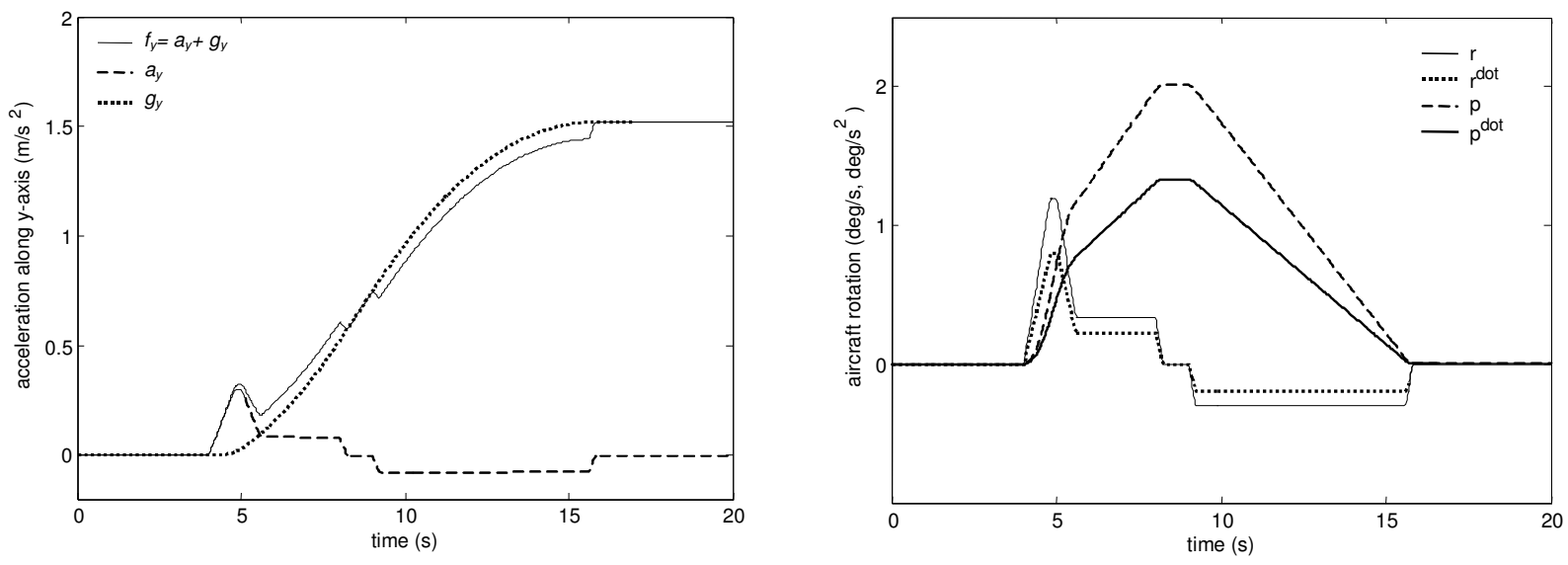

Figure 3. Time history of the output of aircraft model (unfiltered). Left: linear acceleration along the cockpit y-axis. Right: aircraft yaw and roll motion.

Finally, we wished to examine the influence of visual inputs, and for that reason we tested the eight unfiltered motion stimuli under simulated VMC, as well as under simulated IMC. For time considerations, this was not done for Washout ON conditions, which explains the empty box on the bottom right in Figure 4.

All 24 experimental conditions were tested twice: once with crosswind simulated from the right, and once from the left. Airspeed was always $128 \mathrm{kt}$, and cross wind velocity $30 \mathrm{kt}$, corresponding to a slip angle of $13^{\circ}$. Each subject pilot participated in two experimental sessions of 45-60 minutes, separated by a coffee break of 45 minutes. An experimental session was made up by a complete set of 24 conditions in randomized order. However, wind direction was alternated between consecutive trials, meaning that 12 conditions were simulated with right-hand crosswind in the first, and with left-hand crosswind in the second session, and vice versa for the other 12 conditions. 


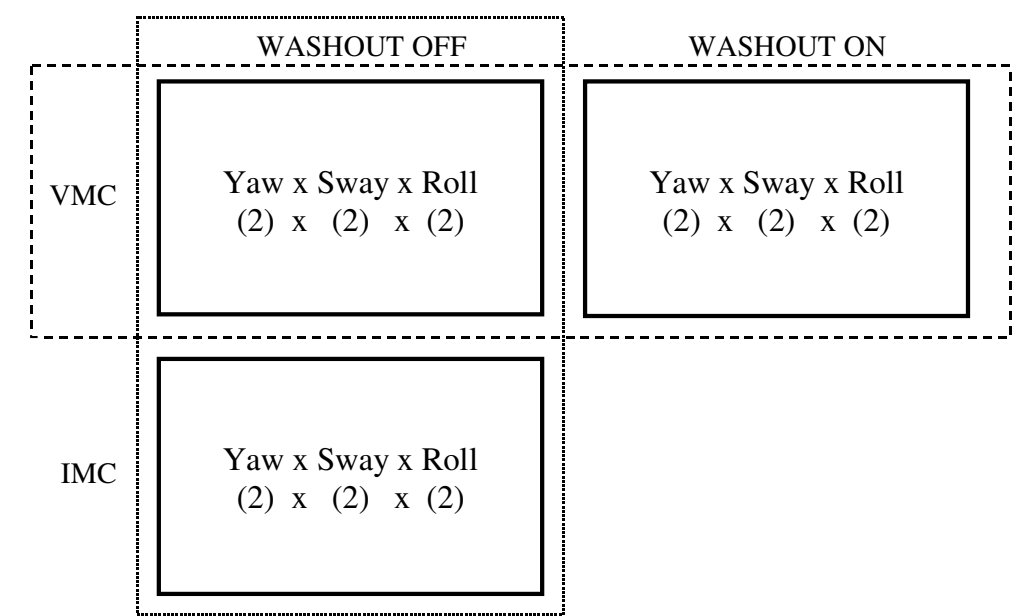

Figure 4. Scheme of experimental design. The dotted and dashed frames indicate the grouping of conditions for the two analyses of variance.

\section{E. Procedure}

Pilots were asked to judge various aspects of motion fidelity. First, they rated the perceived magnitude of simulator motion on a labeled rating scale (Table 1). Although they gave separate ratings for alignment motion (related to the heading change of the aircraft), and roll motion (related to the compensating bank), we will focus here primarily on ratings of the alignment motion, since the combination of yaw, sway and roll in that phase is most interesting in view of the pilot perception model.

The pilots also indicated whether they could differentiate the alignment motion into yaw or sway motion (designated the "motion interpretation task"). Since neither judgement (i.e. subjective magnitude or motion interpretation) did provide information on how realistic the simulation was, we decided to include a four-point scale by which the pilots could express their overall judgement of the entire condition (0="bad"; 1="poor"; 2="good"; 3="excellent").

Table 1. Labeled rating scale to indicate the perceived magnitude.

\begin{tabular}{cl}
\hline Rating & Description \\
\hline 0 & Absent \\
0.5 & Barely noticeable \\
1 & Weak \\
2 & Fairly \\
3 & Clear \\
4 & Strong \\
5 & Very strong \\
\hline
\end{tabular}

\section{Motion perception model}

Figure 5 provides a general outline of the three-dimensional visual-vestibular model used in this study, which was described in more detail by Groen et al. ${ }^{9}$. The model is implemented in Matlab ${ }^{\circledR}$ Simulink $^{\circledR}$, and contains of a set of transfer functions that characterize the primary sensory systems involved in human motion perception: the vestibular system (semicircular canals and otoliths), the visual system (vection), and the somatosensory system. Inter- and intrasensory interactions are also included in the model. Besides on data from the literature, the various parameters have been based on psychophysical studies from our laboratory ${ }^{13-17}$. The six-DoF time histories of the 24 conditions were analyzed off-line with the perception model. An example of this is given in Figure 6 for the perceived lateral acceleration and angle of bank. Platform motion in these motion axes consists of the summed sway and the gravitational component due to tilt. The model output predicts that the specific force $f_{y}$ will be correctly perceived as sway $a_{y}$ in the transient alignment phase (around $\mathrm{t}=5 \mathrm{~s}$ ), and as angle-of-bank $\varphi$ in the stabilized roll phase. Moreover, the effect of modeled visual cues is primarily to suppress the false sensation of sway (Fig. 6, left plot), while on the same time to enhance the perceived angle-of-bank (Fig.6, right plot). 


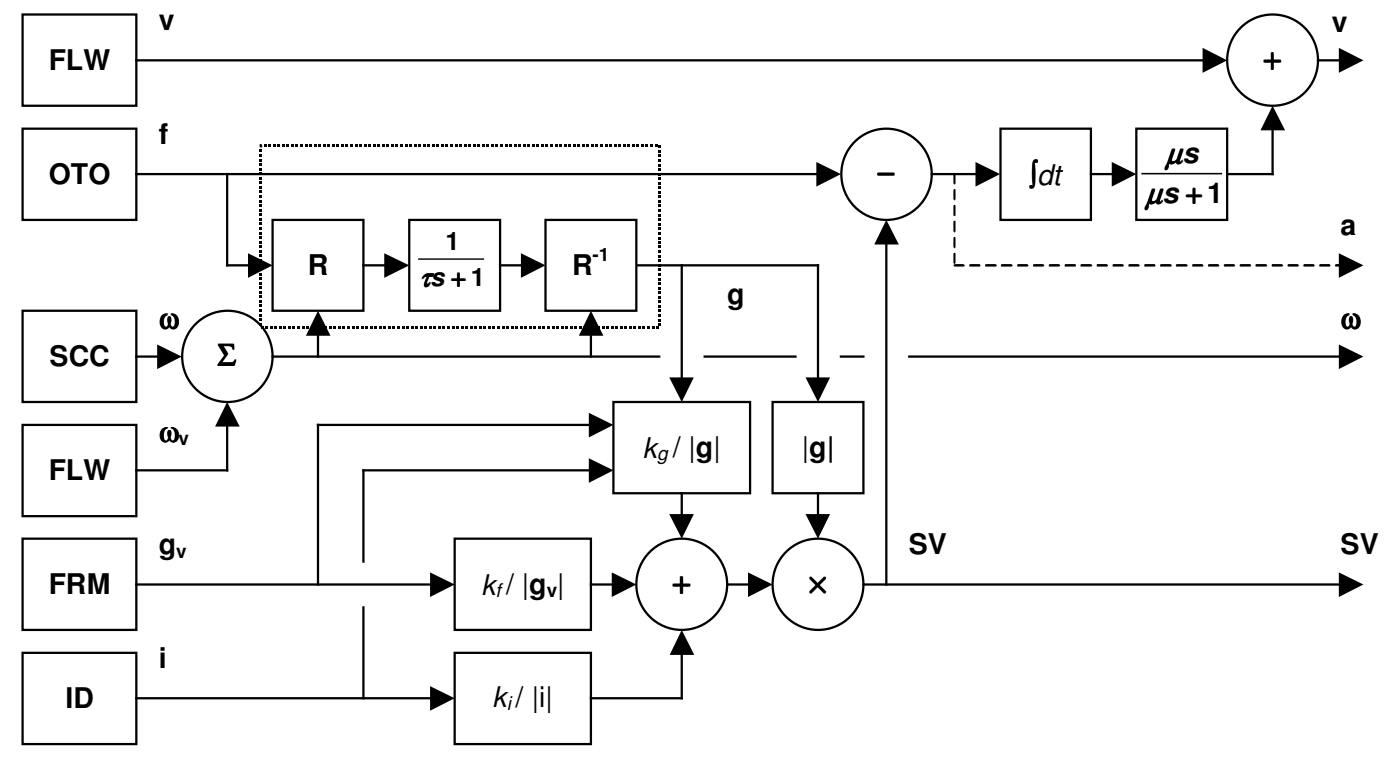

Figure 5. Outline of the sensory interactions in the TNO Motion Perception model, describing the neural resolution of the sensed specific force (f) into the perception of the subjective vertical (SV), linear acceleration (a), angular $(\omega)$ and linear velocity (v). FLW=optic flow; OTO=otoliths; SCC=semicircular canals; FRM=visual frame; ID=ideotropic vector. For explanation see: Groen et al. 2004.
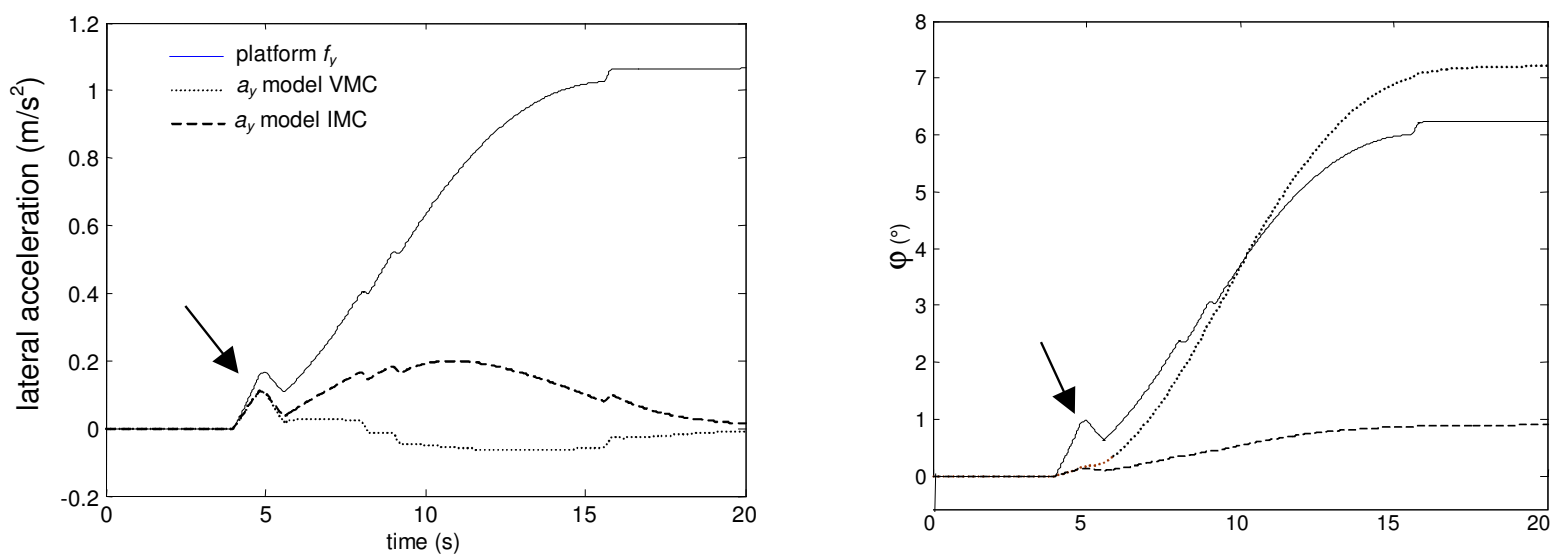

Figure 6. Time histories of model-predicted perception of lateral acceleration (left) and angle of bank (right) in the unfiltered motion condition with visual information (VMC) and without (IMC).

Since the model output produces time histories of angular or linear motion, some extra processing was required for comparison with the pilots' magnitude ratings. To this end, the maximum values of the model-predicted yaw rate and sway acceleration were computed for the phase where aircraft's yaw acceleration peaked (which according to [1] coincides with maximum sway acceleration). This is shown by the arrows in Figure 6. The thus obtained maximum values for yaw and sway were normalized into equivalent units by converting them into multiples of the corresponding perceptual threshold (i.e. $0.5 \%$ for yaw, and $0.1 \mathrm{~m} / \mathrm{s}^{2}$ for sway). Assuming additive effects of yaw and sway cues on the perceived magnitude, both normalized values were then summed into one single estimate. The results of this model-predicted magnitude estimate are shown in Figure 7. 


\section{Results}

\section{A. Data analysis}

The first hypothesis was tested by analyzing the subjective magnitude ratings using a within-subjects Analysis of Variance, or ANOVA. Since the experimental design was not completely symmetrical (the Washout ON conditions were only tested in VMC, not in IMC), we carried out two different analyses. One analysis concerned all VMC conditions testing the factors: Washout $(2) \times$ Roll (2) $\times$ Yaw (2) $\times$ Sway (2). The other analysis concerned all unfiltered motion conditions, testing the factors Visual (2) $\times$ Roll $(2) \times$ Yaw (2) $\times$ Sway (2). Furthermore, the second hypothesis was examined by comparing the correlation between the model output and the subjective pilot data on one hand, with the correlation between platform motion and the pilot data on the other hand. Because the multiple motion parameters involved, we used a multiple regression analysis with the independent variables: platform sway (acceleration); yaw (acceleration, rate); roll (acceleration, rate, bank angle); and visual roll rate (visual sway was considered negligible). For one regression analysis these independent variables were derived from the model output, and for the other analysis the variables were taken from the stimulus. Both regression analyses were performed twice: once with the pilots' magnitude rating as dependent variable, and once with the pilots' motion interpretation as dependent variable. In all analyses, effects with $p<0.05$ were considered significant.

\section{B. Magnitude ratings}

For technical reasons the data set of the first two pilots was incomplete, and their data was discarded for analysis. Moreover, due to a non-linearity in one of the actuators, a false cue (bump) was noticeable at the beginning of Washout OFF trials with sway motion starting in the outmost left position (i.e. with right-hand crosswind). Sway motion starting from the right was unaffected by this, and we decided to use only trials with left-hand crosswind for the analysis.

The ANOVA on the magnitude rating of the alignment phase showed main effects for the following factors: Washout $(\mathrm{F}(1,10)=10.1)$, with unfiltered motion rated stronger than filtered motion; Visual information $(\mathrm{F}(1,10)=26.8)$, with simulated VMC producing higher magnitude ratings than IMC; Sway motion $(\mathrm{F}(1,10)=30.6)$, with sway motion producing stronger motion sensations; and Roll motion $(\mathrm{F}(1,10)=27.5)$, which also positively affected the alignment phase. Remarkably, yaw motion did not produce a main effect, but resulted in a two-way interaction $(F(1,10)=22.3)$ with sway, indicating that platform yaw only contributed to the pilots' judgements in the absence of platform sway. Figure 8 shows the mean magnitude ratings of nine pilots (note that the layout of the figure was chosen to match that of the experimental design in Figure 4, not to suggest a three-way interaction).

The magnitude ratings of the roll phase (not shown in a figure) were positively affected by platform roll and visual cues (VMC). Platform yaw resulted in a small increase, but only in VMC. The factors platform sway and Washout had no structural effect.

\section{Overall judgement}

The mean overall judgements of the entire decrab maneuver, comprising both the alignment and the roll phase, are depicted in Figure 9. According to the ANOVA, overall judgements improved significantly with platform roll (mean increase from 1.1 to 1.6), and also with yaw (increase from 1.2 to 1.5). In addition, there was a main effect for Washout $(\mathrm{F}(1,10)=8.8)$, with Washout ON conditions rated better (mean of 1.4) than Washout OFF conditions (mean of 1.1). A significant two-way interaction between Washout $\times$ Sway $(F(1,10)=6.9)$ indicates that, although filtered sway motion did improve the mean overall judgement (increase from 1.33 to 1.58), unfiltered sway motion had a negative effect on pilots' judgements (decrease from 1.36 to 1.08). According to the pilots' comments, platform motion felt too strong in the Washout ON conditions that contained sway. The opposite was observed for roll motion, where a significant two-way interaction Washout $\times$ Roll $(F(1,10)=5.4)$ indicated that unfiltered roll motion was appreciated best.

\section{Motion interpretation}

The pilots mentioned that it was often difficult to decide whether platform motion during the alignment phase consisted of pure yaw, sway, or a combination of both. In fact, two pilots could not make any differentiation between yaw and sway, and said that it just felt like "alignment motion". Figure 10 depicts the percentage of cases where platform motion was interpreted as sway, yaw, or a combination of both. Although the data sample is too small for statistical analysis, a few interesting observations can be made. First, in most conditions all three categories (yaw, sway, or both) were represented, underlining the difficulty of recognizing the correct platform motion. Second, the fixed-base motion condition ("y0s0r0") also resulted in the perception of some kind of 

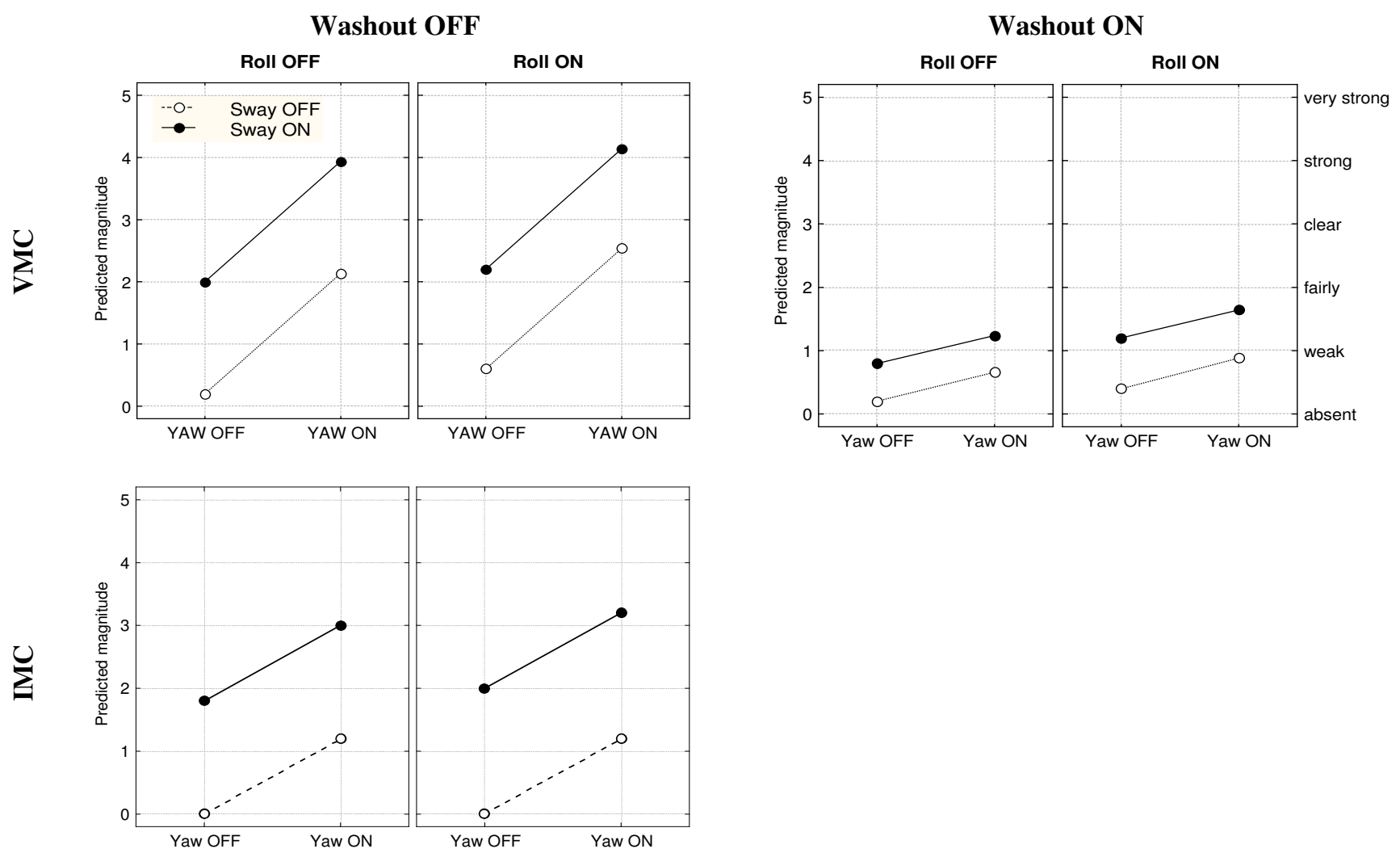

Figure 7. Model-predicted magnitude ratings for alignment motion.
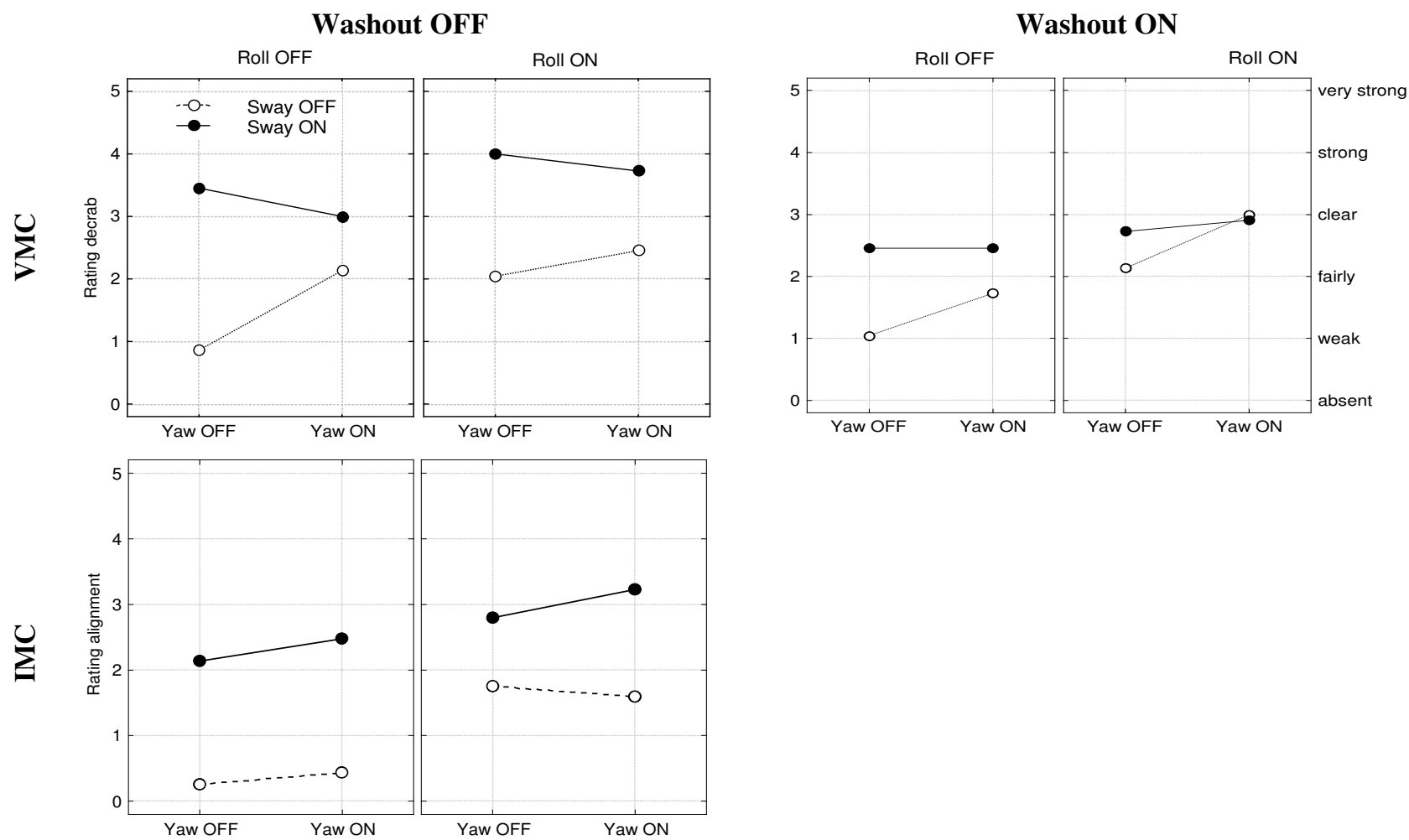

Figure 8. Mean pilots' magnitude rating for alignment motion. 

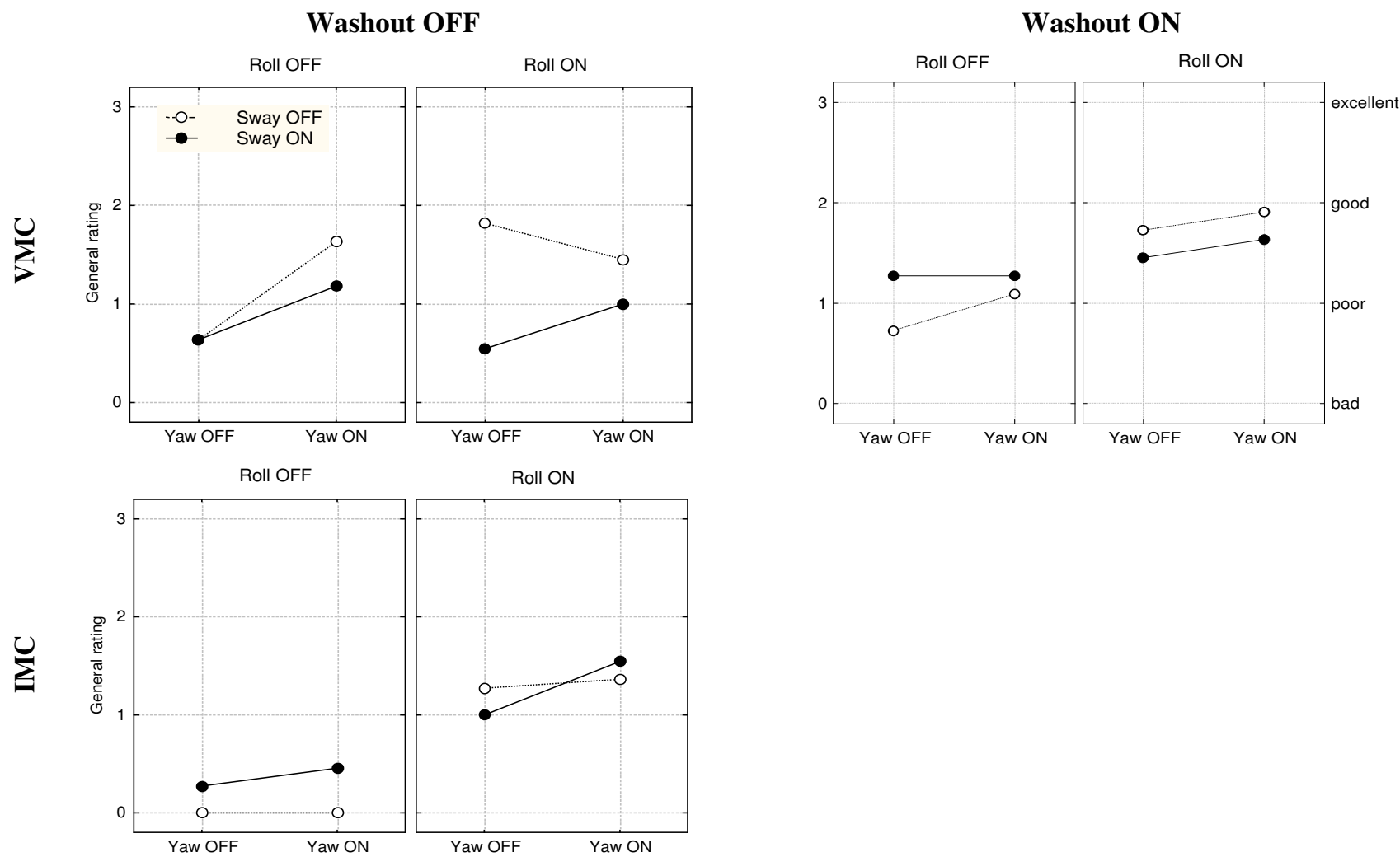

Figure 9. Mean overall judgement of entire decrab maneuver.

Washout OFF
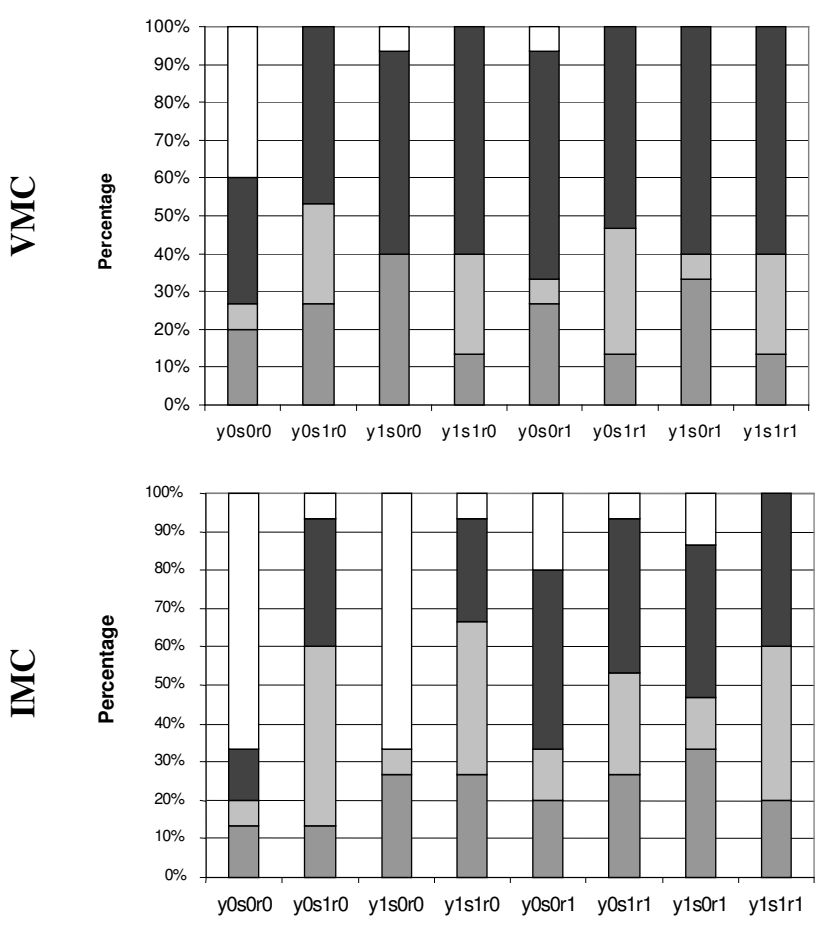

Washout ON

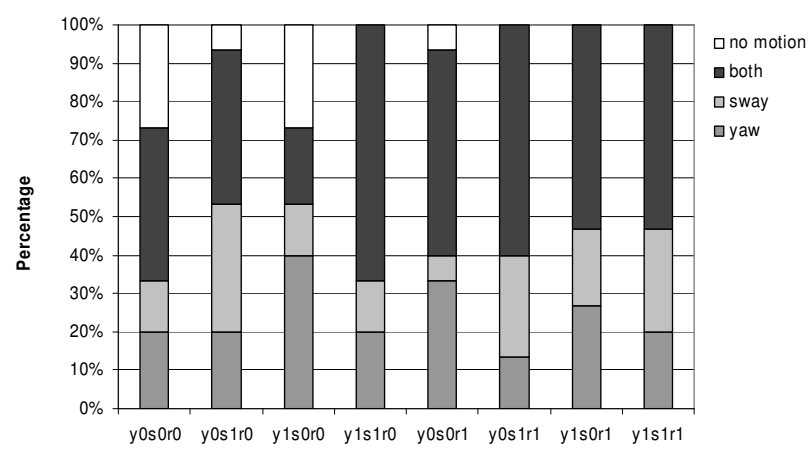

y0 = yaw off, y1= yaw on

s0 = sway off, s1 = sway on

$\mathrm{r} 0=$ roll off, $\quad \mathrm{r} 1=$ roll on

Figure 10. Results of the motion interpretation task, showing percentages of cases in which platform motion in the alignment phase was perceived as yaw, sway, both yaw and sway, or no motion. 
alignment motion. In the VMC condition, almost $75 \%$ of the conditions were perceived as alignment motion. In contrast, in the IMC this percentage was about 33\%, showing the effectiveness of a visual display to induce vection. Still, the fact that the no-motion condition in IMC, i.e. without inertial or visual cues, also induced perceived selfmotion, was rather unexpected. Finally, the third observation is that pure roll motion ("y0s0r1") was often perceived as heading alignment.

\section{E. Correlation with model output}

Comparison of figures 7 and 8 clearly shows that the model-predicted magnitude values did not produce the same pattern as the actual pilot ratings. Indeed, multiple regression analysis on the magnitude ratings showed no difference in correlation coefficients between model output and stimulus parameters (i.e. the actual amplitudes of the sway, yaw , and roll motion cues). The correlation coefficients were $\mathrm{R}^{2}=0.45$ and $\mathrm{R}^{2}=0.47$, respectively.

With respect to the motion interpretation task, on the other hand, multiple regression analysis showed a significantly better correlation between the model output and the total percentage of cases in which platform motion was interpreted as alignment motion (i.e. the summed scores of yaw, sway, and both), than the correlation between this summed score and the stimulus values.

$$
\begin{array}{lll}
\text { Stimulus: } & \mathrm{y}=55-0.6 p_{\text {visual }}-0.7 a_{y}+0.5 p-0.4 a_{y} p & \mathrm{R}^{2}=0.79 \\
\text { Model: } & \mathrm{y}=34-0.8 p_{\text {visual }}-1.2 a_{y}+0.9 p-1.1 a_{y} p & \mathrm{R}^{2}=0.99
\end{array}
$$

The variables in the regression lines indicate that the subjective ratings were significantly determined by visual motion, platform sway, platform roll, and an interaction between sway and roll. This confirms the results of the ANOVA from the previous section, but in a more quantitative manner.

\section{Discussion}

\section{A. Simulation of decrab maneuver}

According to the subjective data, the simulation of heading alignment during the decrab maneuver largely depended on platform sway and roll, rather than yaw. The small effect of yaw was overshadowed in the presence of platform sway, even though the amplitude of both cues was about the same with respect to the respective perceptual threshold. Hence, we must reject our first hypothesis, which stated that yaw and sway motion would provide similar motion feedback to the pilot in this asymmetric flight maneuver. Although our results apply to pilots who did not have an active control task, it is not unlikely that sway motion is also the most critical motion cue in other asymmetric flight maneuvers, such as an engine failure. In fact, in the particular case of an engine failure, pilots do not initiate the motion themselves, but "observe" the motion in much the same way as in the present study. This is of interest, because the unexpected nature of an engine failure makes it a difficult subject for psychophysical studies, which require repeated exposures.

The positive effect of platform roll that we found can be attributed to the gravitational component along the pilot's y-axis that results from platform tilt. With the correct visual stimulus, this gravitational component is interpreted by the pilot as lateral acceleration instead of tilt. This is shown by the model output in Figure 6, where the acceleration along the pilot's y-axis (i.e. the summed sway acceleration and the gravitational component) is interpreted almost entirely as sway motion during the alignment phase, but aircraft bank in the final phase of the decrab. This also explains the interaction term (i.e. sway $\mathrm{x}$ roll) found in the multiple regression analysis.

In addition to sway and roll motion, visual motion cues (in the VMC conditions) also improved the pilots' judgements of the simulation. Remarkably, the results of the motion interpretation task (Figure 10) showed that about 30\% of the pilots still perceived some alignment motion in the IMC condition without platform motion (i.e. fixed base). According to Figure 8 the average magnitude was almost zero ("just noticeable"), but the fact that pilots reported some alignment motion indicates that they sometimes perceive the type of motion that they expect. This effect will even be more candid in man-in-the-loop situations, where the pilot actively controls the simulator and thus expects certain motion feedback. The phenomenon that the human brain sometimes "fills in" missing information is one of the most difficult aspects to capture in perception modeling, but internal models offer a valuable approach in this respect ${ }^{7}$.

An interesting element in this study was the use of unfiltered platform motion. By pre-positioning the simulator, the linear travel of the motion platform approximated that of the actual aircraft. The only difference was a downscale factor of 0.7 , necessary to stay within the actuator linear workspace. The advantage of bypassing the washout filters is that the temporal behavior of the motion profile was not affected by the filter dynamics. On average, the 
magnitude of sway motion was rated stronger in the Washout OFF conditions ("strong") than in the Washout ON conditions ("clear). Notably, the overall judgements show that pilots preferred filtered sway motion, because they considered unfiltered sway motion as too strong. Although pilots were free to choose their own reference for the magnitude rating, most of them explained afterwards to draw a line between the labels "clear" and "strong", where a rating "strong" was given to indicate that the motion sensation was stronger than expected. The result that the sway stimulus with a gain of 0.7 was judged unacceptably strong, confirms previous findings that with the simulation of linear accelerations inertial motion cues are structurally overestimated in a simulator environment ${ }^{10,17,18}$. This phenomenon is not found for angular motion ${ }^{11,19}$. Where in previous studies the dynamics of the washout filter may have had an unknown contribution to this effect, in the present study we can be sure that it is the amplitude, rather than the temporal behavior of the linear motion cue which is overestimated.

\section{B. Model validation}

Practical validation of a motion perception model is complicated by the fact that the pilots' perception can only be measured indirectly through psychophysical procedures. In contrast, the validation of a pilot control model can make use of more quantitative measures (e.g. RMS values of control input). This does not disqualify motion perception models for the evaluation of simulator motion cues: the two types of models are complementary, and relate to different aspects of motion fidelity. A pilot control model may indicate whether platform motion (negatively) affects the closed-loop control task. However, it is possible that a pilot perceives inadequate platform motion, without a marked effect on his control input. In fact, control behavior and perception can be regarded as different levels of a nested control loop: the inner loop relating to attitude control, and the outer loop relating to situational awareness ${ }^{20}$.

Another difficulty with motion perception research is, that the motion stimulus should be exactly defined. This is clearly not the case when the pilot is actively controlling the simulator. This was one of the reasons to have the pilots judge the platform motion as pilots non-flying. In order to make the model output quantitatively comparable to the subjective pilot ratings, we took the maximum value of the model-predicted time histories of perceived yaw and sway motion, and computed these values into an arbitrary magnitude value, based on multiples of the tentative perceptual threshold.

The correlation analysis between the model and subjective data only yielded a positive result for the motion interpretation task. Perceived magnitude, however, was not predicted better by the model than by the stimulus, as we had hypothesized. This was due to the two-way interaction between yaw and sway as observed in the subjective data, which was not reflected in the model output. As shown in Figure 7, the model suggested additive effects for these motion cues. The current state of the model includes various inter-sensory interactions. For example, the model accounts for interactions between the vestibular and visual system (optic flow) in the perception of linear and angular motion. It also accounts for the effects of visual frame on the perception of attitude. The implemented interactions primarily relate to the neural discrimination of self-motion and self-tilt ${ }^{7}$. Accordingly, the model was used successfully to explain the observed pilot ratings of tilt co-ordination in the simulation of sustained linear acceleration, which employs the ambiguity between tilt and translation ${ }^{9}$. Likewise, in the present study the model did predict a positive contribution of visual information (vection) and platform roll to judgements of the alignment phase, which explains the positive correlation between model output and the motion interpretation task. However, the motion interpretation task had a rather qualitative character, and the discrepancy between the model and the observed magnitude ratings points out that our understanding of possible interactions between inertial cues is still insufficient for quantitative evaluation. To some extent, the non-linear interaction between yaw and sway may be caused by differences in perceptual thresholds. Perceptual thresholds strongly depend on the context, illumination and on the duration that a motion cue is sustained ${ }^{21,22}$. Often, perceptual thresholds are determined in isolation (i.e. in the absence of any other motion cue), and in the dark. Here we applied yaw and sway motion simultaneously, and in a dynamic visual environment. We believe that motion cueing in flight simulators, and also our motion perception model, will benefit significantly from better knowledge of relevant perceptual thresholds of motion cues that act congruently (such as yaw and sway in this study), or incongruently (e.g. false cues).

Our ambition in this area is to integrate the motion perception model with our pilot control model to allow for complete off-line evaluation of new motion drive algorithms, for example in our Desdemona simulator (see also presentation of Wentink et al., this conference ${ }^{23}$ ). 


\section{Conclusions}

We conclude that the most effective motion feedback for the simulation of the heading alignment during a decrab maneuver consists of platform sway and roll, not yaw. Visual motion cues also contribute in a positive way. To achieve realistic simulation, the sway motion cue should be considerably smaller than that of the actual aircraft. In this sense, the downscale factor inherent to washout algorithms has a beneficial side effect for the simulation of linear accelerations.

With respect to the motion perception model we conclude that the model does not yet allow for exact quantitative evaluation of the effectiveness of ground-based motion cues. One of the reasons for this is that currently no perceptual thresholds have been implemented in the model. The problem is that there is insufficient knowledge in the literature on perceptual thresholds that apply to a flight simulator environment.

\section{Acknowledgments}

This work was supported by the Basic Research Project " 49313 TM", granted by the Netherlands Agency for Aerospace Programmes, NIVR. The authors would like to thank Ir. Jacco Dominicus and Ing. Paul Breed of the NLR for their professional support in setting up the flight simulator; Dr. Jelte Bos of TNO for his expertise on human perception modeling; and finally the Research and Development Department of Rexroth-Hydraudyne Inc. for sharing their thoughts on motion-critical flight maneuvers.

\section{References}

${ }^{1}$ Chung, W.Y., "A review of approaches to determine the effectiveness of ground-based flight simulation," AIAA Modeling and Simulation Technologies Conference, A00-37311, 2000.

${ }^{2}$ Hosman, R. J. A. W., "Are criteria for motion cueing and time delays possible?", AIAA Modeling and Simulation Technologies Conference, CP-99-4028, 1999.

${ }^{3}$ Hosman, R. J. A. W., "Pilot's perception and control of aircraft motions," Ph.D. Dissertation, Control and Simulation Department, Delft Technical University, The Netherlands, 1996.

${ }^{4}$ Sivan, R., Ish-Shalom, J., and J. K. Huang "An optimal approach to the design of moving flight simulators", IEEE Transactions on Systems, Man and Cybernetics, Vol. 12, No. 6, 1982, pp. 818-827.

${ }^{5}$ Bussolari, S. R., Sullivan, R. B., and Young, L. R., "Vestibular models for the design and evaluation of simulator motion", Proceedings of the RAeS Conference on: "Advances in flight simulation - Visual and motion systems", 1986.

${ }^{6}$ Telban, R. J., Cardullo, F. M., and Houck, J. A., "Developments in human centered cueing algorithms for control of flight simulator motion systems," CP-99-4328, AIAA Modeling and Simulation Technologies Conference, 1999.

${ }^{7}$ Bos, J. E., and Bles, W., "Theoretical considerations on canal-otolith interaction and an observer model," Biological Cybernetics, Vol. 86, 2002, pp.191-207.

${ }^{8}$ Bos, J. E., Bles, W., and Hosman, R. J. A. W., "Modeling human spatial orientation and motion perception," AIAA Modeling and Simulation Technologies Conference, CP-2001-4248, 2001.

${ }^{9}$ Groen E. L., Hosman, R. J. A. W., Bos, J. E., and Dominicus, J. W., "Motion perception modelling in flight simulation," CP-13, Flight Simulation 1929-2029: A centennial perspective”, Royal Aeronautical Society, 2004.

${ }^{10}$ Groen, E. L., Valenti Clari, M. S. V., and Hosman, R.J.A.W., "Evaluation of perceived motion during a simulated takeoff run," Journal of Aircraft, Vol. 38, No. 4, 2001, pp. 600-606.

${ }^{11}$ Groen, E. L., Hosman R. J. A. W., and Dominicus J. W., "Motion fidelity during a simulated takeoff," AIAA Modeling and Simulation Technologies Conference, CP-2003-5680, 2003

${ }^{12}$ Borah, J., Young, L. R., and Curry, R. E., "Sensory mechanism modeling," AFHRL-TR-78-83, Air Force Human Resources Laboratory, Brooks AFB, TX, 1979.

${ }^{13}$ Groen, E. L., Howard, I. P., and Cheung, B. S. K., "Influence of body roll on visually induced sensations of self-tilt and rotation," Perception, Vol. 28, 1999, 287-297.

${ }^{14}$ Bos, J. E., Cheung, B. S. K., and Groen, E. L., "The somatogravic effect without concomitant angular motion," TNO-report TM-01-002, TNO Human Factors, Soesterberg, the Netherlands, 2001.

${ }^{15}$ De Graaf, B., Bos, J. E., Tielemans, W., Rameckers, F., Rupert, A.H., and Guedry, F. E., "Otolith contribution to ocular torsion and spatial orientation during acceleration," NAMRL Technical Memorandum 96-3, Naval Aerospace Medical Laboratory, Pensacola (Fl)

${ }^{16}$ Groen E. L., Jenkin, H. L., and Howard, I. P., "Perception of self-tilt in a true and illusory vertical plane," Perception, Vol. 31, 2002, pp. 1477-1490.

${ }^{17}$ Groen E. L., and Bles, W., "How to use body tilt for the simulation of linear self motion," Journal of Vestibular Research, Vol. 14, 2004, pp.375-385.

${ }^{18}$ Harris, L. R., Jenkin, M., and Zikovitz, D. C., Visual and non-visual cues in the perception of linear self-motion," Experimental Brain Research, Vol. 135, 2000, pp.12-21

${ }^{19}$ Van der Steen, F. A. M., "An earth-stationary perceived visual scene during roll and yaw motions in a flight simulator," Journal of Vestibular Research, Vol. 8, No. 6, 1998, pp. 411-425. 
${ }^{20}$ Hosman R. J. A. W., Hamman B., Pelchat, Y., and Schroeder J., "Summary of the panel discussion on motion cueing requirements," AIAA Modeling and Simulation Technologies Conference, CP-2001-5586, 2001.

${ }^{21}$ Berthoz, A., Pavard, B., Young, L. R., "Perception of linear horizontal self-motion induced by peripheral vision (linearvection)," Experimental Brain Research, Vol. 23, 1975, pp.471-489.

${ }^{22}$ Huang, J., and and Young, L. R., "Sensation of rotation about a vertical axis with a fixed visual field in different illuminations and in the dark," Experimental Brain Research, Vol. 41, 1981, pp.172-183.

${ }^{23}$ Wentink, M., Mayrhofer, M., Hosman, R.J.A.W., and Bles, W., "Design and evaluation of spherical washout algorithm for Desdemona simulator", AIAA Modeling and Simulation Technologies Conference, 2005. 